\title{
Selection Of Spectrum Sensing Method To ENHANCE QOS IN COGNITIVE RADIO NETWORKS
}

\author{
Nabil Giweli, Seyed Shahrestani and Hon Cheung \\ School of Computing, Engineering and Mathematics, Western Sydney University, \\ Sydney, Australia
}

\begin{abstract}
The massively increasing number of wireless communication devices has led to considerable growths in radio traffic density, resulting in a predictable shortage of the available spectrum. To address this potential shortage, the Cognitive Radio (CR) technology offers promising solutions that aim to improve the spectrum utilization. The operation of $C R$ relies on detecting the so-called spectrum holes, i.e., the frequency bands when they are unoccupied by their licensed operators. The unlicensed users are then allowed to communicate using these spectrum holes. Consequently, the performance of CR is highly dependent on the employed spectrum sensing methods. Several sensing methods are already available or literarily proposed. However, no individual method can accommodate all possible CR operation scenarios. Hence, it is fair to ascertain that the performance of a CR device can be improved if it is capable of supporting several sensing methods. Then it should be able to effectively select the most suitable method. In this paper, several spectrum sensing methods are compared and analyzed, aiming to identify their advantages and shortcomings in different CR operating conditions. Furthermore, it identifies the factors that need to be considered while selecting a proper sensing method from the catalog of available methods.
\end{abstract}

\section{KEYWORDS}

Cognitive Radio; Spectrum Sensing; QoS

\section{INTRODUCTION}

Conventionally, the Radio Frequency Spectrum (RFS) is statically regulated into licensed and unlicensed bands. While the use of the former is restricted to authorized operators, the unlicensed bands are available for use by the public subject to transmission constraints [1]. Under such regulation, the unlicensed bands may get heavily congested. On the other hand, several studies and measurements conducted around the world have indicated that the licensed RFS bands can be underutilized [2].

From a technical perspective, the Cognitive Radio (CR) concept is a promising technology to achieve an efficient utilization of RFS. The concept for CR model was introduced in 1999 by Joseph Mitola [3]. In these models, the operators licensed to use some particular frequency bands are considered as the Primary Users (PUs), whereas, the unlicensed participants are referred to as the Secondary Users (SUs). The CR model is based on the realization that a PU may not fully utilize its licensed bands, leaving parts of its spectrum unoccupied. These unoccupied white spaces, or holes, relate to use, or more correctly the lack of use, in terms of frequency, time, or space and location. An SU can utilize these holes in addition to the unlicensed bands that it may typically use. 


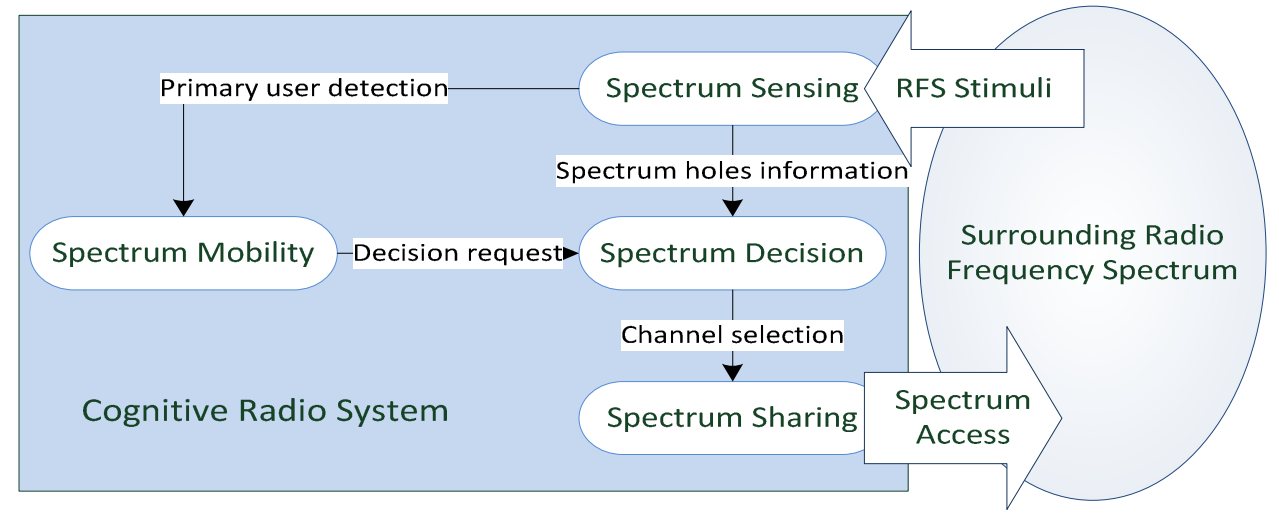

Figure 1. Basic CR cycle

To achieve their objectives to maximize the utilization of the available frequency spectrum, CR systems are dependent on the execution of a sequence of several functions, the so-called CR cycle. A typical CR cycle was proposed by Mitola [3]. This is illustrated in Figure 1.

The main functions of the CR cycle are spectrum sensing, spectrum decision, spectrum sharing, and spectrum mobility. More specifically, an SU should be able to perform the following [4]:

- Spectrum sensing: sense the surrounding RFS to determine spectrum holes and to detect the presence of the relevant PU.

- Spectrum decision: analyze and decide which spectrum hole is the most suitable for satisfying the application requirements.

- Spectrum sharing: share the available spectrum holes with other SUs as fairly as possible.

- Spectrum mobility: seamlessly switch to another suitable spectrum hole to avoid interference with a detected PU that may wish to start using its licensed spectrum. The decision of which spectrum hole is a suitable for handoff to is to be requested from spectrum decision function.

Detecting the presence of a PU, or more precisely finding out whether the PU is using its allocated spectrum or not, is an essential task for a CR device. On one hand, this fundamental task requires improving sensing accuracy by avoiding false positive results while detecting the presence of a PU. On the other, the employed sensing technique should achieve a high detecting probability of the available spectrum holes. The nature of the electromagnetic signals makes accurate sensing a complicated process. More specifically, the Signal to Noise Ratio (SNR), the multipath fading of the PU signals, and the changing levels of noise can significantly affect the sensing accuracy [5, 6]. Moreover, imperfect spectrum sensing can result in increased transmission error rates, for both the PU and the SUs [7]. Such errors may contribute to the degradation of the quality of services (QoSs) provided by a PU and SUs. Noticeably, any QoS degradation that can be attributed to the CR technology can potentially harm the progress of the CR-based solutions. In this paper, the main features and limitations of the prevalent spectrum sensing methods are examined. Furthermore, the key aspects that should be involved in selecting the appropriate sensing method are highlighted and discussed.

The remainder of this paper is organized as follows. Section II presents the background and motivation for this work. The effects of the sensing operation on the QoS of the applications running over CR networks are described in Section III. Several sensing approaches are studied and discussed in Section IV. Factors that may help in selecting the proper sensing techniques are outlined in Section V. The last section gives the conclusions and points to the potential future expansion of the reported work. 
International Journal of Wireless \& Mobile Networks (IJWMN) Vol. 8, No. 1, February 2016

\section{Motivations}

Most of the previous reviews of spectrum sensing techniques are mainly focused on the operation, accuracies, complexities, and implementation issues [8-11]. For instance, the relation between the sensing accuracy and the sensing time or frequency is the primary focus of the authors in [8]. They suggested that the focus should be on finding an optimal spectrum sensing technique with the capability of flexible tuning between time and frequency resolutions. For such optimization possibility, they nominate a wavelet-based spectrum estimation method. However, they find that the available state-of-art sensing technologies do not offer a possible trade-off between the complexity of the sensing method and its accuracy. In contrast, other reviewers consider the characteristics of the PU signal as the main factor for selecting a proper sensing method [9]. Nevertheless, other factors should be considered for more adaptive sensing and improved performance.

In general, the common approach is about how to find an optimal sensing method for all possible $\mathrm{CR}$ operation requirements. However, all the aforementioned references agree that none of the proposed sensing methods is suitable for all possible sensing situations, conditions and technologies of CR systems. This study shifts the focus to a new approach where a CR device supports a range of various sensing methods. Thus, the proper sensing technique can be selected based on the real-time requirements. This approach implies the need for a real-time mechanism to select the most suitable sensing method based on a number of factors or criteria. In this paper, various sensing methods are studied toward finding the relevant selection criteria that should be considered when designing such as real-time selection mechanism.

\section{Sensing Operation Impact on Applications' QOS IN CR NETWORKS}

As shown in Figure 2, the sensing operation of a CR consists of repeated cycles of the sensing period $\mathrm{T}$. The sensing period effectively represents the time interval where sensing is repeated together with data transmission. It also represents the communication frame in CR, referred to as a CR frame. During the sensing time $t$ in a sensing period, a CR device obtains frequency spectrum information, called RFS Stimuli in Figure 1, from its environment of surrounding radio frequency spectrum. After the sensing time, the CR device can decide to transmit data on the same channel or in a new vacant channel, i.e., a spectrum hole, based on the outcome from the spectrum decision component.

The decision taken by the CR device is based on the sensing outcome, i.e., the presence or absence of suitable spectrum holes as well as that of the PU. The data transmission starts after the sensing time until the next sensing period, also called the CR communication frame. The data transmission time (T-t) depends on the sensing time $t$ and the $\mathrm{CR}$ frame time $\mathrm{T}$ and hence the data transmission is based on how frequent and long the sensing is conducted. Thus, sensing frequency is $1 / \mathrm{T}$. The sensing time $t$ and the $\mathrm{CR}$ frame time $\mathrm{T}$ can be designed to be fixed for all frames or could be designed to vary based on the design goals [12]. Typically, sensing should be as short and less frequent as possible without affecting the sensing accuracy [13]. 


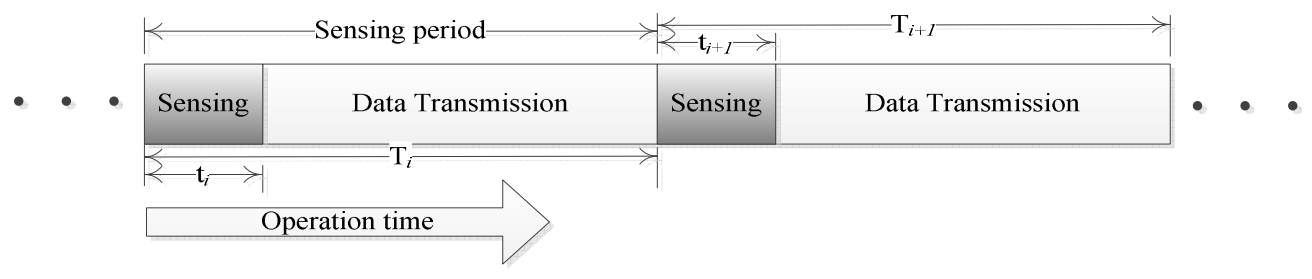

Figure 2. Simple structure of CR frames based on sensing operation

Increasing the sensing time $t$ and conducting sensing more frequently, i.e. decreasing $\mathrm{T}$, lead to an increase in the probability of correct detection of the PU's presence. In turn, this leads to more protection to the PU from interference by other CR users and more utilization of the spectrum. On the other hand, it leads to less data transmission rate and hence to QoS degradation for SUs. The degradation can be measured by several parameters such as throughput, delay and MAC layer process overhead [14]. Therefore, designing the sensing time and frequency of sensing should take into account the trade-off between protecting the PU's QoS and improving the QoS of SUs.

\section{SENSING METHODS}

The main challenge facing the sensing methods is how to improve the spectrum sensing performance by mainly increasing the positive detection probability and decreasing the false detection probability. A sensing technique with a higher positive detection probability provides more protection to PU. A CR device with a lower probability of false detection of the presence of the PU has more chance to use the available spectrum holes. Therefore, the CR device has more chance of achieving a higher throughput on the CR network. The design of a sensing technique is constrained by an acceptable level of false detection [15]. Additionally, improving sensing performance is challenged by a range of trade-offs and various constraints such as application requirements, hardware capability, complexity and required infrastructure [16].

In general, a sensing method that uses surrounding RFS information collected by the CR device only is called a local sensing method. If the SUs do not exchange their surrounding RFS information gathered by local sensing, then the sensing method is referred to as a non-cooperative sensing method. In this paper, the sensing methods are also classified into three categories: methods with no prior sensing information required, methods based on prior sensing information and methods based on sharing of sensing information amongst SUs.

\subsection{Blind Sensing}

In blind sensing, no prior information about the PUs' signal is necessary for the sensing methods under this category. However, prior information about the noise power of the targeted spectrum may be required for better performance. Otherwise, a reasonable estimation of the noise power is used instead. Two well-known blind sensing methods are based on energy detection and covariance-based detection.

\subsubsection{Energy Detection}

The energy detection, also known as radiometry or periodogram, is the most common method for spectrum sensing because of its low implementation complexity and computational overhead [5]. In this method, an energy detector is used to monitor the energy level of the communication channel with a narrowband frequency spectrum and then the observed signal energy level is compared with a predefined threshold. The channel is occupied by the PU if the signal's energy is 
over the threshold; otherwise, it is considered unoccupied and therefore a spectrum hole exists in the channel. Because of this simplicity, this technique requires the shortest sensing time t per CR frame compared to other common sensing technologies [17].

Generalizing the use of this method faces several challenges as a consequence of its simplicity. Firstly, selection of the threshold used for detection becomes an issue when the channel noise level is unknown or uncertain over time [18]. Secondly, under a low SNR, it is hard to differentiate between modulated signals, including signals of other SUs, noise, and interference, resulting in poor detection performance [5]. Lastly, an energy detector is ineffective in detecting spread spectrum signals [19].

\subsubsection{Covariance-based Detection}

This method is based on comparing the covariance of the observed signal and the covariance of the noise where statistical covariance matrices of signal and noise are usually different [20]. The main improvement of this method is to overcome the energy detection shortcomings. In particular, it can distinguish between signal and noise in a low SNR, and without any prior information about the PU's signal and channel noise. This improvement is achieved at the expense of adding a computational overhead in computing the covariance matrix of the observed signal samples [11]. In addition to an increase in complexity, other drawbacks of the energy detection are still present in the covariance-based detection.

The covariance-based detection method works with no prior information about the PU signals. They have a limited performance particularly when the PU uses spread spectrum signals for its transmission techniques. Moreover, the blind sensing methods could not effectively distinguish between PU and other SUs that are sharing the same spectrum holes. Research is ongoing to improve the blind sensing approach in terms of performance and required sensing time, such as in $[21,22]$.

\subsection{Prior Information Required}

Methods belonging to this category rely on partial or full information about the PU's transmission signal to be able to differentiate it from other signals and noise. In this category, the sensing method takes several samples of the surrounding spectrum of a certain channel and extracts any possible features of the collected samples. Then it compares these characteristics with the prior known features of the PU signal. The algorithms used to derive and compare these features differ from sensing method to another. Also, the amount and type of the required prior information are based on the used method. In this section, several of the sensing methods belong to this category are described.

\subsubsection{Cyclostationarity Feature Detection}

This method is based on distinguishing the PU signal from noise, interference, and other signals by identifying its cyclostationarity features [23, 24]. These cyclostationarity features are associated with the signal modulation type, carrier frequency, and data rate. Hence, the CR device needs sufficient prior information about these unique characteristics of the PU signal. Based on this information, it can perform a cyclostationarity analysis on the signal to identify matched features [9]. For this method to perform better than the energy detection method, an adequate number of real-time sample sets in the frequency domain need to be collected. As a consequence, better performance accrues more complexity and sensing time at the expense of the available throughput [9]. 
International Journal of Wireless \& Mobile Networks (IJWMN) Vol. 8, No. 1, February 2016

\subsubsection{Correlation Detection}

Sensing based on correlation is also known as waveform-based sensing or coherent sensing. In this method, the expected correlation or coherence between the observed signal samples is identified to detect the PU signal based on previous knowledge about its waveform patterns [9]. The accuracy of the sensing increases when the length of the known signal pattern of the PU is increased [25]. The main drawback of this method is related to the large amount of information required for signal patterns of the PUs to achieve a high performance that is not practical for several CR systems.

\subsubsection{Radio Identification Based Sensing}

This method is based on having a priori information about the transmission technologies used by the PU. In the radio identification stage of the method, several extracted features of the received signal are exploited and then classified to determine if the signal demonstrates the PU signal technology [26]. Fundamentally, the feature extraction and classification techniques are used in the context of the European Transparent Ubiquitous Terminal (TRUST) project [27]. For collecting and extracting the signal features, the radio identification method may use one of the known sensing techniques, such as the energy detection method [9]. The radio identification improves the accuracy of the energy detection to some extent with complexity implication. The achieved precision is dependent on the signal feature extraction and classification techniques used to identify the presence of the PU.

\subsubsection{Matched Filtering}

The matched filtering method achieves a higher detection probability in a shorter detection time, compared to other methods that are similarly based on prior information [28, 29]. This method is considered as the best sensing method in this classification. The collected signal is passed through a filter that will amplify the possible PU signal and attenuate any noise signal. The filter makes the detection of the presence of the PU signal more accurate [29]. The filter, which is known as a matched filter, has to be tuned based on some features of the PU signal. These features include the required bandwidth, operating frequency, the modulation used and frame format [9]. One of the disadvantages of this method is in implementation where different PUs signal types require different dedicated hardware receivers. This requirement makes the method difficult to be practically implemented and also leads to higher power consumption during operation if the method is implemented based on current hardware technologies.

Figure 3 shows an estimated comparison between non-cooperative sensing methods, based on accuracy and complexity of the reviewed sensing methods in this study. Table 1 shows more comparison aspects between local sensing methods. The comparison clearly shows that a higher accuracy is achieved in the cost of complexity. Moreover, sensing methods with higher performance and more robustness against noise require more sensing time and sufficient prior information about the PU signal. 


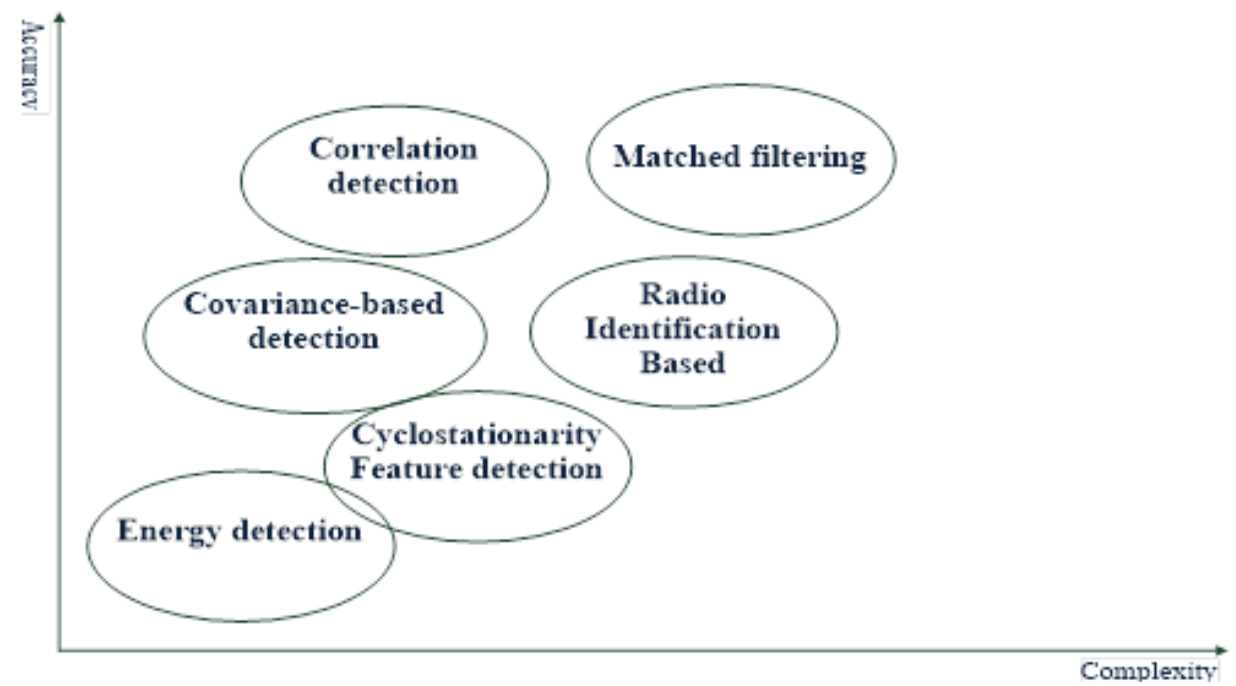

\begin{tabular}{|l|l|l|l|l|l|}
\hline Sensing Method & $\begin{array}{l}\text { Sensing } \\
\text { Time }\end{array}$ & $\begin{array}{l}\text { Robustness } \\
\text { Against } \\
\text { Noise }\end{array}$ & $\begin{array}{l}\text { Detection } \\
\text { Performance }\end{array}$ & Complexity & $\begin{array}{l}\text { Prior } \\
\text { Information } \\
\text { Required }\end{array}$ \\
\hline Matched filter & High & High & High & High & High \\
\hline $\begin{array}{l}\text { Radio Identification } \\
\text { Based }\end{array}$ & Medium & Medium & Medium & High & Medium \\
\hline Correlation & High & High & Medium & Medium & Medium \\
\hline $\begin{array}{l}\text { Cyclostationarity } \\
\text { Feature detection }\end{array}$ & High & High & Low & Medium & High \\
\hline Covariance & Medium & Medium & Low & Medium & None \\
\hline Energy detection & Low & Low & Low & Low & Low \\
\hline
\end{tabular}

Table 1. Comparison between local sensing methods

\subsection{Based on SU Cooperation}

The main principle of this approach is that SUs share their locally sensed information of the spectrum with each other. The use of sensed information from other SUs can produce a more accurate sensing outcome than relying solely on local sensing. The hidden transmitter problem is an example of the issues that may prohibit a CR from detecting the presence of a PU, when the $\mathrm{CR}$ relies only on locally sensed information. The cause of this problem is the fading and shadowing of the signals emitted from a PU, although it is within the transmission range of the CR [9]. However, when cooperated SUs are spatially distributed, it helps to overcome the hidden PU problem and other limitations of local sensing [30]. Sensing cooperation can also reduce the local sensing cost, e.g., sensing time and energy consumption while maintaining sensing quality by scheduling the sensing operation among cooperative SUs [31]. The sensing method used by an individual SU can be based on one of the sensing methods for local sensing, such as energy detection and cyclostationarity feature detection [10]. 
In some environments, cooperative sensing may lose its advantages as far as an individual SU is concerned. For instance, increasing the local sensing frequency in an individual SU of high mobility is more efficient, in terms of sensing accuracy and overhead, than to cooperate with other SUs [19]. In cooperative sensing, the improvement of sensing is more noticeable when the number of cooperative SUs is increased. However, involvement of more SUs will increase the cooperation overhead in terms of the amount of data exchange and the time required for the exchange [32].

A cooperative approach can only be used when SUs are able and willing to collaborate. Also, a SU may not always find other cooperative SUs within its transmission range. Therefore, the CR devices should not solely rely on cooperative sensing approaches. They should be able to use a suitable local sensing method and resort to cooperative sensing, only when an enhanced performance is possible.

\section{Factors for Selecting Suitable Sensing Method}

Selecting the suitable sensing method for a particular CR operation condition depends on several factors. These factors may change during the CR operation. Thus, the proper sensing method for the new conditions might be a different method. Based on the discussions in previous sections, notable factors are described in the following sub-sections.

\subsection{Applications QoS Requirements}

The QoS requirements differ based on the applications running on a CR device. The sensing delay and transmission throughput vary from one sensing method to another within the same operating conditions. As a result, the sensing operation used on a CR device has a direct impact on the QoS of an application running on the device, mainly in terms of the throughput, delay and jitter. As sensing is a repetitive operation, a CR device should be able to select a proper sensing method with the least impact on the QoS of the running application. As shown in Table 1, the required sensing time and the accuracy level vary depending on the sensing method used. Using a sensing method having a higher sensing accuracy leads to greater utilization of the spectrum. However, achieving a higher sensing accuracy requires more sensing time that causes more delay to the running application. For example, the sensing time for energy detection is usually less than $1 \mathrm{~ms}$ while cyclostationarity feature detection requires $24 \mathrm{~ms}$ or more [17]. For an optimal sensing accuracy, the sensing time needs to be in the range of 2 seconds as has been found in [33]. Such a long sensing time is not suitable for delay sensitive applications, such as VoIP. Hence, the sensing methods that require long sensing times should be avoided when a CR device is running an application sensitive to delay. Similarly, other operational requirements must also be taken into account.

\subsection{Availability of Prior Information}

The amount of information available about the characteristics of the PUs and the communications media is an important factor influencing the selection of a proper sensing method. For instance, insufficient information about the PU signals excludes the use of the methods utilizing matched filters. To achieve an optimal sensing accuracy, a CR device should be able to change the sensing method based on the information that becomes available about the PU signal or the SNR of the targeted spectrum. The energy detection method performs more effectively when the SNR is high so it could be the preferred sensing method in this situation and this method has a minimal overhead in terms of sensing time and power consumption. In contrast, in low or uncertain SNR, the energy detection method performs very poorly and thus should be avoided. Under the absence 
of any prior information about the PU, the selection of the sensing method is limited to the blind sensing methods. The range of the sensing methods based on prior information that can be used is depended on the type of the available information about the PU. For example, the transmission technology used by a PU is enough for sensing methods based on radio identification, but it is not adequate for those based on the use of matched filters, as they requires more detailed information. Obtaining very specific information about a PU, including the modulation used and frequency operation, is not possible in some cases. The operation of a CR device usually involves switching amongst different frequency bands licenced to different PUs. Therefore, the CR device has to change the sensing method based on the information available about the new frequency band.

\subsection{Level of Protection Required for $P U$}

The selection of the sensing method must be considered with regard to the degree of protection necessary for the PU. They may vary depending on available frequency bands and types of services. For instance, analog TV service is more robust against interference than digital TV service [16]. Hence, a sensing method that provides less protection, i.e., lower PU detection probability, should only be used when the PU is more tolerant of interference such as in analog TV services. The PU application sensitivity should also be considered on the protection level required. For example, PU signals used for military and security purposes require a high level of protection. High accuracy sensing may be enforced in frequency bands where the PU signals used for critical missions. This factor poses the need of classifying different protection levels for PUs based on the transmission technology being used by the PUs and their application sensitivity. These protection levels can be assigned to each licensed band and announced. Currently, the IEEE standardization effort, for CR, specifies only one general protection level, i.e., the minimum accepted level of detection probability is $90 \%$ [34]. The wide adoption of the CR technology is based on how extent this technology success in guaranteeing an adequate level of protection to the PUs.

\subsection{CR Device Capability}

A CR device designed with limited hardware resources and power capacities will not be able to support a wider range of sensing methods. Some methods require sophisticated hardware components and higher power consumptions, e.g. the matched filter method, compared to simple ones such as the energy detection method. An ideal CR device should be able to be reconfigured on-the-fly to support a broad range of sensing methods. In practice, a CR device's actual capability will limit the range of sensing methods that can be supported. For mobile CR devices, which is capable of supporting a wide range of sensing methods, the remaining battery power or the economic power consumption theme can be considered in the selection of a sensing method requiring lower power consumption.

\subsection{The CR Network Mode and Capability}

The network mode and capability are important factors to CR systems to make a decision between cooperative and non-cooperative sensing approaches. In CR networks with infrastructure and centralized topology, a method based on cooperative sensing is more suitable than that based on local sensing only. Hence, the capability of such a CR network depends on how much management ability can provide for white space determination to its CR devices. Furthermore, the capacity of a CR network relies on how much information the network can gather and provide to its CR users about the PU signals and the ambient spectrum. 
In addition, the Medium Access Control (MAC) techniques used by a CR device should be considered in selecting a local sensing method. In general, the MAC techniques can be classified into three approaches: random access, time slotted and hybrid protocols [35, 36]. The Carrier Sense Multiple Access with Collision Avoidance (CSMA/CA) mechanism is widely used in random access and hybrid based protocols. The well-known IEEE 802.11stadnrads are based on CSMA/CA where the random access or hybrid based protocols are adopted. When a CR device uses the CSMA/CA to content on the same spectrum holes, the sensing operation will be conducted more often. Hence, the selection of the sensing method should be considered under two stages. The first stage of sensing is conducted to avoid interference with the PU user. While the second stage is performed within the contention window to avoid interference with other CR users. In the first stage, as the PU protection is the main concern, a high accuracy method should be used. In the second stage, the energy detection method is usually used because it requires a small sensing time. In the time slotted approach, the CR users share the spectrum based on Time-Division Multiple Access (TDMA) which avoids interference between CR users [37]. Hence, the sensing operation is only needed to protect the PU. Therefore, TDMA networks require sensing less frequent than random access networks. The hybrid protocols are used to achieve a compromisation between random access and time slotted approaches. This compromisation should be also reflected on compromising switching between complex and simple sensing methods.

\section{Conclusions}

The work reported in this paper indicates that none of the available spectrum sensing techniques can achieve perfect detection of spectrum holes or the presence of the PU for all potential CR operating conditions. The spectrum sensing operation has an essential impact on the QoS of the running applications and overall performance in CR. This impact varies based on the sensing method used. Therefore, to improve the performance of CR systems, the relevant devices must be capable of utilizing a range of sensing methods. The selection of the most suitable method from the available sensing methods is based on a number of factors that have also been identified and discussed in this paper. The two most important factors among them are the QoS requirements and the required protection level for PU signals. This study poses the need of a real-time selection mechanism of the proper sensing method with the consideration of the stated factors. Our future works will focus on more exhaustive evaluations of these factors and how their fine-tunings can contribute to an improved CR performance. Furthermore, a real-time mechanism for selecting the proper sensing method based on these factors is our future aim.

\section{REFERENCES}

[1] L. Cui and M. B. Weiss, "Can unlicensed bands be used by unlicensed usage," Paper for the 41st annual TPRC, September, pp. 27-29, 2013.

[2] K. Patil, R. Prasad, and K. Skouby, "A Survey of Worldwide Spectrum Occupancy Measurement Campaigns for Cognitive Radio," in Devices and Communications (ICDeCom), 2011 International Conference on, 2011, pp. 1-5.

[3] J. Mitola and G. Q. Maguire Jr, "Cognitive radio: making software radios more personal," Personal Communications, IEEE, vol. 6, pp. 13-18, 1999.

[4] J. Marinho and E. Monteiro, "Cognitive radio: survey on communication protocols, spectrum decision issues, and future research directions," Wireless networks, vol. 18, pp. 147-164, 2012/02/01 2012.

[5] Y. Zeng, Y.-C. Liang, A. T. Hoang, and R. Zhang, "A review on spectrum sensing for cognitive radio: challenges and solutions," EURASIP J. Adv. Signal Process, vol. 2010, pp. 2-2, 2010.

[6] A. F. Molisch, L. J. Greenstein, and M. Shafi, "Propagation issues for cognitive radio," Proceedings of the IEEE, vol. 97, pp. 787-804, 2009. 
[7] S. Haddadi, H. Saeedi, and K. Navaie, "Channel coding adoption versus increasing sensing time in secondary service to manage the effect of imperfect spectrum sensing in cognitive radio networks," in Communication and Information Theory (IWCIT), 2013 Iran Workshop on, 2013, pp. 1-5.

[8] D. Ariananda, M. Lakshmanan, and H. Nikookar, "A survey on spectrum sensing techniques for cognitive radio," in Cognitive Radio and Advanced Spectrum Management, 2009. CogART 2009. Second International Workshop on, 2009, pp. 74-79.

[9] T. Yucek and H. Arslan, "A survey of spectrum sensing algorithms for cognitive radio applications," Communications Surveys \& Tutorials, IEEE, vol. 11, pp. 116-130, 2009.

[10] I. F. Akyildiz, B. F. Lo, and R. Balakrishnan, "Cooperative spectrum sensing in cognitive radio networks: A survey," Physical Communication, vol. 4, pp. 40-62, 2011.

[11] D. B. Rawat and G. Yan, "Spectrum sensing methods and dynamic spectrum sharing in cognitive radio networks: A survey," International Journal of Research and Reviews in Wireless Sensor Networks, vol. 1, pp. 1-13, 2011.

[12] P. Yiyang, L. Ying-Chang, K. C. Teh, and L. Kwok Hung, "How much time is needed for wideband spectrum sensing?," Wireless Communications, IEEE Transactions on, vol. 8, pp. 5466-5471, 2009.

[13] C. Kae Won, "Adaptive Sensing Technique to Maximize Spectrum Utilization in Cognitive Radio," Vehicular Technology, IEEE Transactions on, vol. 59, pp. 992-998, 2010.

[14] H. Xin-Lin, W. Gang, H. Fei, and S. Kumar, "The Impact of Spectrum Sensing Frequency and PacketLoading Scheme on Multimedia Transmission Over Cognitive Radio Networks," Multimedia, IEEE Transactions on, vol. 13, pp. 748-761, 2011.

[15] L. Ying-Chang, Z. Yonghong, E. C. Y. Peh, and H. Anh Tuan, "Sensing-Throughput Tradeoff for Cognitive Radio Networks," Wireless Communications, IEEE Transactions on, vol. 7, pp. 1326-1337, 2008.

[16] A. Ghasemi and E. S. Sousa, "Spectrum sensing in cognitive radio networks: requirements, challenges and design trade-offs," Communications Magazine, IEEE, vol. 46, pp. 32-39, 2008.

[17] H. Kim and K. G. Shin, "In-band spectrum sensing in cognitive radio networks: energy detection or feature detection?," in Proceedings of the 14th ACM international conference on Mobile computing and networking, 2008, pp. 14-25.

[18] R. Tandra and A. Sahai, "SNR Walls for Signal Detection," Selected Topics in Signal Processing, IEEE Journal of, vol. 2, pp. 4-17, 2008.

[19] D. Cabric, S. M. Mishra, and R. W. Brodersen, "Implementation issues in spectrum sensing for cognitive radios," in Signals, Systems and Computers, 2004. Conference Record of the Thirty-Eighth Asilomar Conference on, 2004, pp. 772-776 Vol.1.

[20] Y. Zeng and Y.-C. Liang, "Spectrum-sensing algorithms for cognitive radio based on statistical covariances," Vehicular Technology, IEEE Transactions on, vol. 58, pp. 1804-1815, 2009.

[21] Y. Li, S. Shen, and Q. Wang, "A Blind Detection Algorithm Utilizing Statistical Covariance in Cognitive Radio," International Journal of Computer Science Issues (IJCSI), vol. 9, pp. 7-12, 2012.

[22] K. S. Kumar, R. Saravanan, and R. Muthaiah, "Cognitive Radio Spectrum Sensing Algorithms based on Eigenvalue and Covariance methods," International Journal of Engineering \& Technology (09754024), vol. 5, 2013.

[23] J. Lundén, V. Koivunen, A. Huttunen, and H. V. Poor, "Spectrum sensing in cognitive radios based on multiple cyclic frequencies," in Cognitive Radio Oriented Wireless Networks and Communications, 2007. CrownCom 2007. 2nd International Conference on, 2007, pp. 37-43.

[24] S. Enserink and D. Cochran, "A cyclostationary feature detector," in Signals, Systems and Computers, 1994. 1994 Conference Record of the Twenty-Eighth Asilomar Conference on, 1994, pp. 806-810.

[25] H. Tang, "Some physical layer issues of wide-band cognitive radio systems," in New frontiers in dynamic spectrum access networks, 2005. DySPAN 2005. 2005 first IEEE international symposium on, 2005, pp. 151-159.

[26] T. Yucek and H. Arslan, "Spectrum characterization for opportunistic cognitive radio systems," in Military Communications Conference, 2006. MILCOM 2006. IEEE, 2006, pp. 1-6.

[27] T. Farnham, G. Clemo, R. Haines, E. Seidel, A. Benamar, S. Billington, et al., "Ist-trust: A perspective on the reconfiguration of future mobile terminals using software download," in Personal, Indoor and Mobile Radio Communications, 2000. PIMRC 2000. The 11th IEEE International Symposium on, 2000, pp. 1054-1059.

[28] S. Shobana, R. Saravanan, and R. Muthaiah, "Optimal Spectrum Sensing Approach on Cognitive Radio Systems," 2013. 
[29] S. Shobana, R. Saravanan, and R. Muthaiah, "Matched Filter Based Spectrum Sensing on Cognitive Radio for OFDM WLANs," International Journal of Engineering and Technology (IJET), vol. 5, 2013.

[30] W. Chien-Min, S. Hui-Kai, L. Maw-Lin, L. Yi-Ching, and L. Chih-Pin, "Cooperative Power and Contention Control MAC Protocol in Multichannel Cognitive Radio Ad Hoc Networks," in Innovative Mobile and Internet Services in Ubiquitous Computing (IMIS), 2014 Eighth International Conference on, 2014, pp. 305-309.

[31] X. Dongyue, E. Ekici, and M. C. Vuran, "Cooperative Spectrum Sensing in Cognitive Radio Networks Using Multidimensional Correlations," Wireless Communications, IEEE Transactions on, vol. 13, pp. 1832-1843, 2014.

[32] Z. Yulong, Y. Yu-Dong, and Z. Baoyu, "Cooperative relay techniques for cognitive radio systems: Spectrum sensing and secondary user transmissions," Communications Magazine, IEEE, vol. 50, pp. 98-103, 2012.

[33] S. RAJPUT, N. BOHRA, H. M. HARIS, P. KHALID, and S. Z. SHAH, "A Modified Energy Detection Based Spectrum Sensing Algorithm for Green Cognitive Radio Communication," Mehran University Research Journal of Engineering \& Technology, vol. 34, 2015.

[34] "ISO/IEC/IEEE International Standard -- Information technology -- Telecommunications and information exchange between systems -- Local and metropolitan area networks -- Specific requirements -- Part 11: Wireless LAN medium access control (MAC) and physical layer (PHY) specifications AMENDMENT 5," ISO/IEC/IEEE 8802-11:2012/Amd.5:2015(E) (Adoption of IEEE Std 802.11af-2014), pp. 1-204, 2015.

[35] C. Cormio and K. R. Chowdhury, "A survey on MAC protocols for cognitive radio networks," Ad Hoc Networks, vol. 7, pp. 1315-1329, 2009.

[36] I. F. Akyildiz, W.-Y. Lee, and K. R. Chowdhury, "CRAHNs: Cognitive radio ad hoc networks," Ad Hoc Networks, vol. 7, pp. 810-836, 2009.

[37] P. Ren, Y. Wang, Q. Du, and J. Xu, "A survey on dynamic spectrum access protocols for distributed cognitive wireless networks," EURASIP J. Wireless Comm. and Networking, vol. 2012, p. 60, 2012.

\section{AUTHORS}

Nabil Giweli received the B.Sc. degree in Communication Engineering from Tripoli University, Libya, in 1997, the Master degree in Information and Communication Technology (with the dean medal award) from the Western Sydney University in 2011, and another M.Sc. form the same university in Cloud Security in 2013. Currently, he is a $\mathrm{Ph}$.D. candidate and a casual teacher at the School of Computing, Engineering and Mathematics, Western Sydney University, Australia. His current research area is in Cognitive Radio Technologies.

Dr. Seyed Shahrestani completed his PhD degree in Electrical and Information Engineering at the University of Sydney. He joined Western Sydney University (Western) in 1999, where he is currently a Senior Lecturer. He is also the head of the Networking, Security and Cloud Research (NSCR) group at Western. His main teaching and research interests include: computer networking, management and security of networked systems, analysis, control and management of complex systems, artificial intelligence applications, and health ICT. He is also highly active in higher degree research training supervision, with

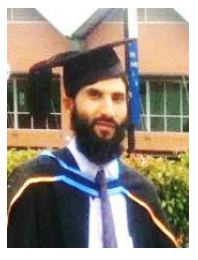
successful results.

Dr. Hon Cheung graduated from The University of Western Australia in 1984 with First Class Honours in Electrical Engineering. He received his $\mathrm{PhD}$ degree from the same university in 1988. He was a lecturer in the Department of Electronic Engineering, Hong Kong Polytechnic from 1988 to 1990. From 1990 to 1999, he was a lecturer in Computer Engineering at Edith Cowan University, Western Australia. He has been a senior lecturer in Computing at Western Sydney University since 2000. Dr Cheung has research experience in a number of areas, including conventional methods in artificial intelligence, fuzzy sets,

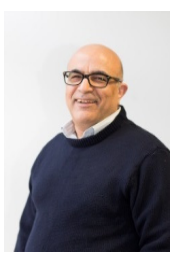
artificial neural networks, digital signal processing, image processing, network security and forensics, and communications and networking. In the area of teaching, Dr Cheung has experience in development and delivery of a relative large number of subjects in computer science, electrical and electronic engineering, computer engineering and networking.

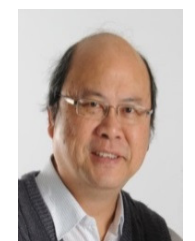

